\title{
Plume Fluxes in Clear and Cloudy Convective Boundary Layers
}

\author{
ULRICH SCHUMANN \\ DLR, Institute of Atmospheric Physics, Oberpfaffenhofen, Germany \\ CHIN-HOH MOENG \\ National Center for Atmospheric Research, Boulder, Colorado
}

(Manuscript received 29 May 1990, in final form 25 February 1991)

\begin{abstract}
From results of large-eddy simulations of the clear convective boundary layer and of a stratus-topped boundary layer, mean properties of "plumes" that consist of "updrafts" and "downdrafts" are determined. The plumes are defined locally by the sign of the vertical velocity or of moisture fluctuation or by a combination of both. As a further alternative, updrafts and downdrafts in which the vertical velocity magnitude exceeds certain threshold values are considered. The first two variants divide the motion field into two streams, whereas in the other variants "environmental" air forms a separate stream. The computed mean properties are in general agreement with existing measurements. From the results we compute mean vertical fluxes assuming "top-hat profiles" and compare these with the actual fluxes. It is shown that the most uniform flux approximation is obtained if the plume structure is classified in terms of vertical velocity $w$. For such " $w$ plumes," the top-hat profiles approximate about $60 \%$ of the actual fluxes if updrafts and downdrafts are distinguished with zero threshold values just according to the sign of the vertical velocity. A higher percentage is obtained with nonzero threshold values.
\end{abstract}

\section{Introduction}

The motion in clear or stratus-cloud topped convective boundary layers exhibits a coherent structure of convective circulations composed of "updrafts" and "downdrafts," possibly separated by "environmental air." The updrafts are composed of quasi-steady largescale plumes, which often extend from the surface far up into the boundary layer and which carry a large portion of the vertical fluxes within the boundary layer. This justifies the "top-hat approximation" in which vertical fluxes $\overline{w^{\prime} f '}$ are computed as if the vertical velocity $w$ and the transported quantity $f$ at a given height were constant within the two or three regions of motion. Let $\alpha_{p}$ denote the area fraction taken by updrafts (subscript $p=u$ ), downdrafts $(p=d)$, and environment $(p=e)$ at a given height and let $w_{p}$ and $f_{p}$ denote the corresponding mean values of $w$ and $f$, respectively. Then previously proposed approximations (e.g., Betts 1973; Greenhut and Khalsa 1987; Manton 1975; Businger and Oncley 1990) are

$\overline{w^{\prime} f^{\prime}}=\omega^{*}\left(f_{u}-\bar{f}\right)$, where $\bar{f}=\alpha_{u} f_{u}+\alpha_{d} f_{d}+\alpha_{e} f_{e}$,

$$
\overline{w^{\prime} f^{\prime}}=\omega^{* *}\left(f_{u}-f_{d}\right)
$$

Corresponding author address: Dr. U. Schumann, DLR, Institut für Physik der Atmosphäre, W-8031 Oberpfaffenhofen, Germany.

$$
\begin{aligned}
& \overline{w^{\prime} f^{\prime}}=a\left(\alpha_{u} w_{u} f_{u}+\alpha_{d} w_{d} f_{d}+\alpha_{e} w_{e} f_{e}\right), \\
& \overline{w^{\prime} f^{\prime}}=b \sigma_{w}\left(f_{u}-f_{d}\right) .
\end{aligned}
$$

Here, $\omega^{*}$ and $\omega^{* *}$ are "flux velocities," $a$ and $b$ are constant coefficients of order unity, and $\sigma_{w}$ is the heightdependent rms vertical velocity fluctuation. These approximations are applicable if $a$ and $b$ are about constant for all relevant quantities $f$, and if $\omega^{*}$ and $\omega^{* *}$ have universal height-dependent profiles within convective layers, both for clear and cloudy cases. The parameter values depend on the criterion used to discriminate between the two or three regions of motion. In previous studies, the top-hat approximation was applied to scalar fluxes. Its applicability for fluxes of horizontal momentum $(f=u)$ or vertical velocity variance $(f=w)$ has not been tested.

Several experimental studies have considered the mean properties of plumes including the related fluxes. A wide variety of plume definitions has been used in the literature. Lenschow and Stephens (1980), for example, studied the convective boundary layer over the ocean and discriminated "thermals" from environmental air by requiring that the humidity be larger than a certain threshold for a sufficiently large distance within the thermal. Penc and Albrecht (1987) followed Nicholls and LeMone (1980) to investigate solid and broken cloud-topped mixed layers. They defined updrafts as that part of a measured time series having collocated positive values of vertical velocity and hu- 
midity fluctuations. Segments of the time series where both quantities are negative are classified as downdrafts, leaving a remainder of environmental air that belongs to neither of these two classes. Greenhut and Khalsa $(1982,1987)$ identified an updraft (downdraft) in the marine atmospheric boundary layer when the vertical velocity fluctuation is larger (smaller) than a certain threshold value for a minimum time along the flight path of the measuring aircraft. Otherwise they classified the motion as belonging to environmental air. Young (1988a,b) analyzed data from a convective boundary layer over land and defined updrafts and downdrafts in terms of either positive or negative spatially filtered vertical velocity. The filter excludes scales smaller than about one-tenth of the boundary-layer thickness. Further studies have been reviewed in the references just mentioned. Hence, practically every study is based on a different definition of plumes, and thus conclusive comparisons cannot be made between the various studies. Moreover, the measurements apply to either clear or cloudy boundary layers and it is not obvious how the findings depend on the type of boundary layer.

In this paper we present analysis of large-eddy simulation (LES) results applying various types of plume definitions in order to find out for which classification the top-hat profile model gives the most uniform and universal approximation, in which the coefficients are the same for any transported quantity and are simple functions of height or are constant. Our ultimate objective is to set up a predictive model for plume dynamics in stratus-topped boundary layers. Since more data on plumes are available for the clear case, we first compare plume statistics from the LES for the dry convective boundary layer (CBL) with observations and then discuss the stratus-topped boundary layer (STBL). By comparing the results from the two cases we learn about the cloud effects with respect to plume properties.

Large-eddy simulations (see the review by Nieuwstadt 1990) resolve the energy-carrying eddies while modeling subgrid-scale (SGS) contributions. They have been used to identify the spatial structure of plumes in the CBL (Schmidt and Schumann 1989) and in the STBL (Moeng and Schumann 1990). Such simulations with fine resolution have been shown to be very successful in simulating quantitatively the characteristic features of buoyancy-dominated clear boundary layers because the convective circulation with plumes or thermals can be resolved. Relatively few simulations have been performed for stratus-topped boundary layers (Deardorff 1980, Moeng 1986).

For the present study, we use available simulation results, which were obtained before from the LES-code of Moeng $(1984,1986)$. We treat the LES results just as experimental data and assess their validity by comparing to experimental results where possible and by comparing the magnitude of the observed effects relative to SGS contributions. The conditional averaging procedure will be based solely on the resolved field.
Thus, our approach is similar to experimental studies that use filtered fields to classify the plume type or require the indicator function to persist over a certain interval.

\section{The large-eddy simulation data}

The method for the clear convective boundary layer (CBL) and the parameters of the simulations, employing $96^{3}$ grid points, are as described in Moeng (1984) and Moeng and Wyngaard (1989). The (equidistant) grid spacings are $\Delta x=\Delta y=52.1 \mathrm{~m}$ in the horizontal directions and $\Delta z=20.8 \mathrm{~m}$ in the vertical. The flow is driven by a constant geostrophic wind of $10 \mathrm{~m} \mathrm{~s}^{-1}$ in the $x$ direction with a Coriolis frequency of $10^{-4} \mathrm{~s}^{-1}$. The results for the CBL are scaled using the convective velocity scale $w_{*}$ and the temperature scale $T_{*}$, which are based on the surface heat flux, the height of the boundary layer $z_{i}$ (actually the height where the vertical heat flux takes its minimum value), and the buoyancy parameter. The ratio between boundary layer height $z_{i}$ and the Obukhov length $L$ is rather large, $z_{i} / L=-14$. Hence, turbulence in the present case is mainly driven by buoyancy with small contributions from shear (friction velocity $u_{*}=0.589$ $\left.\mathrm{m} \mathrm{s}^{-1}, w_{*}=2.02 \mathrm{~m} \mathrm{~s}^{-1}, z_{i}=1030 \mathrm{~m}\right)$. Figure 1 depicts mean profiles of $u, v$, and $\theta$ in the CBL. We observe the well-mixed part of the CBL with close to uniform potential temperature below the inversion. The strong mixing in this layer is also reflected in the velocity profiles. Rather strong vertical shear appears at the inversion and near the surface. The budget of kinetic energy and the profiles of kinetic energy and vertical velocity variance have been shown in Moeng and Wyngaard (1989). The energy budget shows that, in this case, shear contributes only a little to energy production except near the surface. The large value of the vertical velocity variance within the mixed layer indicates strong updrafts and downdrafts. The kinetic energy in the subgrid scales is small (less than 15\%) in compar-

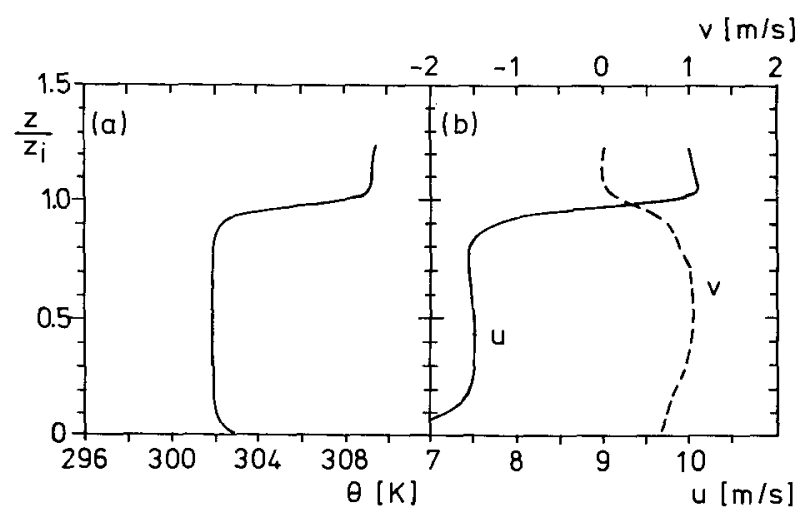

FIG. 1. Mean profiles in the CBL versus height $z$. (a) Potential temperature and (b) horizontal wind components. 
ison to the energy of all scales; i.e., most of the energetic motions are resolved by the LES.

The method for the cloudy case (STBL) and the parameters are as described in Moeng (1986), simulation case I, except that the code was rerun with $80^{3}$ grid points instead of $40^{3}$. The vertical grid width has been halved to $12.5 \mathrm{~m}$. The horizontal grid spacing $(62.5 \mathrm{~m})$ is the same as in Moeng (1986) but the size of the computational domain has been doubled horizontally to cover $5 \mathrm{~km} \times 5 \mathrm{~km} \times 1 \mathrm{~km}$. The larger horizontal domain size allows for the formation of larger scales and reduces statistical uncertainty in approximating ensemble means by horizontal mean values. The surface is kept at constant temperature and at saturation mixing ratio of the moisture. Mean profiles, see Fig. 2, compare closely to previous results (Moeng 1986). The boundary layer possesses a strong inversion at $z_{i}=507 \mathrm{~m}$ below which a 312-m-thick solid layer of stratus cloud forms. The liquid water content grows with height in close agreement with adiabatic mixing. The wind profile is similar to that in the dry case. The inversion extends over only two vertical grid cells. Convection in the mixed layer is driven mainly by both longwave radiation cooling at the top and the surface buoyancy flux as reflected by the total heat flux profile in Fig. $2 d$ and the buoyancy production term $B$, see Fig. 2e, which equals the flux of virtual potential temperature. Here turbulence results are normalized by a convective velocity $w_{*}$, which is based on the averaged buoyancy flux inside the cloud layer (Moeng 1986). Often experimental data have been normalized by the velocity scale

$$
w_{* D}=\left[2.5 T^{-1} g \int_{0}^{z_{i}} \overline{w^{\prime} \theta_{v}^{\prime}} d z\right]^{1 / 3}
$$

introduced by Deardorff (1980), and we use this scale in comparisons. On average, $w_{*}=0.829 \mathrm{~m} \mathrm{~s}^{-1}, w_{* D}$ $=1.102 \mathrm{~m} \mathrm{~s}^{-1}$, and $u_{*}=0.300 \mathrm{~m} \mathrm{~s}^{-1}$. According to the large ratio $z_{i} / L=-20$, shear (curve $S$ in Fig. 2e) contributes significantly only near the surface and, to a minor extent, at the inversion. Turbulent transport (term $D$ in Fig. 2e) balances the energy loss by dissipation $(-\epsilon)$ and buoyancy at the inversion. The profiles of velocity variances and kinetic energy $E$, Fig. 2e, have a shape similar to that in the dry case, except that cloud forcing has caused larger variances in the upper part of the boundary layer.
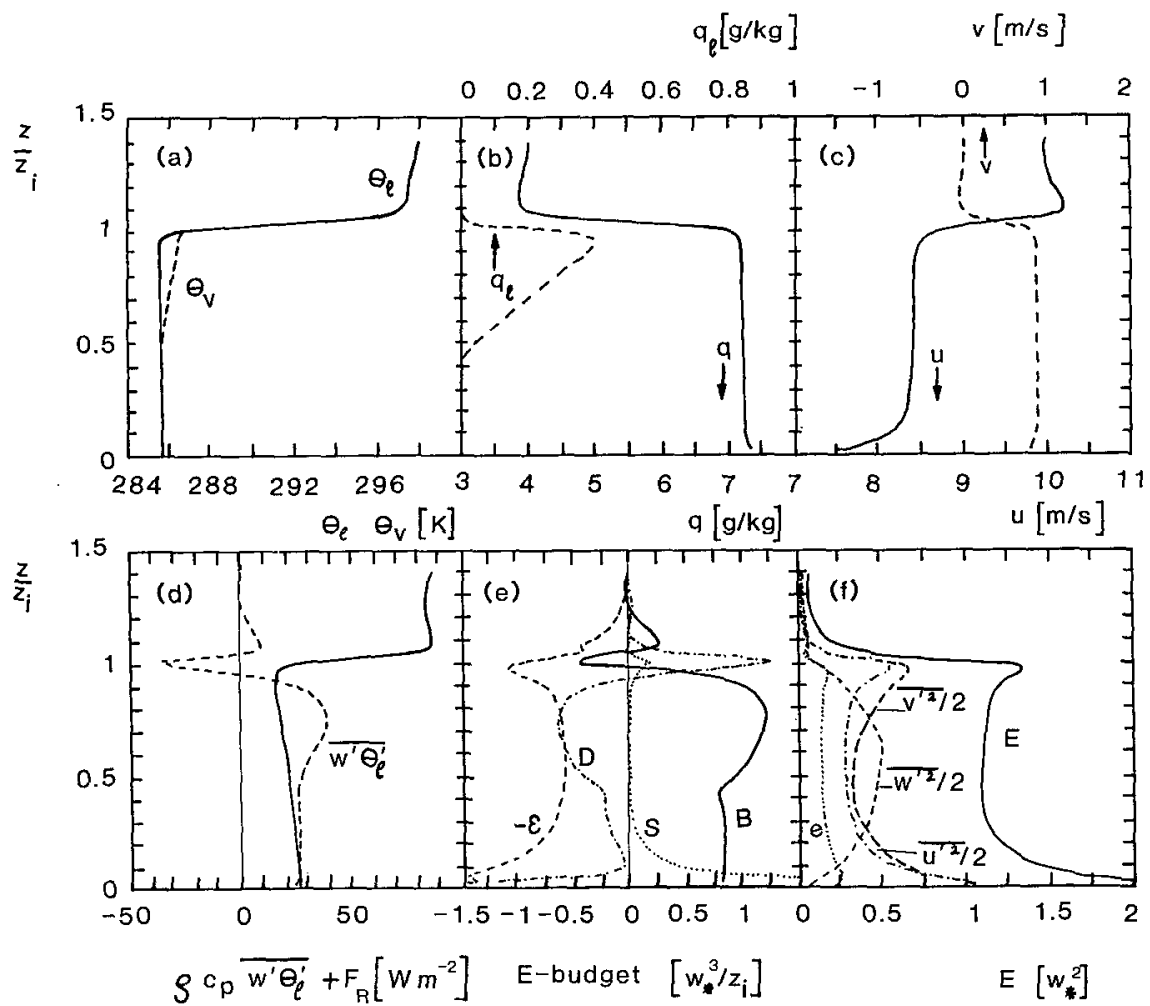

FIG. 2. Mean profiles in the STBL. (a) Liquid water potential temperature (full curve) and virtual potential temperature (dashed); (b) total and liquid water content; (c) wind components; (d) sum of liquid water static energy and longwave radiation fluxes (full curve) and liquid water static energy flux alone (dashed); (e) budget of kinetic energy due to buoyancy $B$, shear $S$, dissipation $\epsilon$, and turbulent transport $D$; and (f) total kinetic turbulence energy $E$, SGS energy $e$, and variance components. 


\section{Conditional sampling method}

We determine conditional mean values $f_{p}$ of any quantity $f$ for either of the three classes $p=u, d, e$ from averages over all discrete grid points, $i=1, \cdots$, $M_{x}, j=1, \cdots, M_{y}$ in a horizontal plane at height $z$ :

$$
\begin{aligned}
f_{p}(z)=\sum_{i=1}^{M_{x}} \sum_{j=1}^{M_{y}} I_{p}\left(x_{i}, y_{j}, z\right) f\left(x_{i}, y_{j}, z\right) / \\
\sum_{i=1}^{M_{x}} \sum_{j=1}^{M_{y}} I_{p}\left(x_{i}, y_{j}, z\right),
\end{aligned}
$$

where $I_{p}$ is the "indicator function" that discriminates between "updrafts" $\left(I_{u}\right)$, "downdrafts" $\left(I_{d}\right)$, and possibly "environmental air" $\left(I_{e}\right)$ through the following definitions:

1) " $w$ plumes" using vertical velocity: $I_{u}(x, y, z, t)$ $=1$ if $w(x, y, z, t)>w_{t h}^{+}, I_{u}=0$ otherwise; $I_{d}(x, y$, $z, t)=1$ if $w(x, y, z, t) \leqslant w_{t h}, I_{d}=0$ otherwise; $I_{e}$ $=1-I_{u}-I_{d}$. In general and for comparison with Young (1988b), we assume zero threshold values $w_{t h}^{+}$ $=w_{t h}^{-}=0$, so that the set of grid points with environmental air is empty. Greenhut and Khalsa $(1982,1987)$ set thresholds for updrafts (downdrafts), $w_{t h}^{+}\left(w_{t h}^{-}\right)$, equal to the square root of one-half the variance of all positive (negative) values of $w^{\prime}$ about $w^{\prime}=0$. In comparing to their measurements we find better agreement, however, when omitting the factor one-half (i.e., using $\sqrt{2}$ larger threshold values), as will be explained below. In order to compare with Nicholls (1989), who investigated strong downdrafts using a nonlocal criterium, we will present results with $w_{t h}^{-}=0$ and with $w_{t h}^{-}$ $=-0.5 w_{* D}$.

2) " $q$ plumes" like $w$ plumes but using total moisture (vapor plus liquid water concentration) instead of vertical velocity and zero threshold values.

3) " $w q$ plumes" using vertical velocity combined with total moisture: $I_{u}(x, y, z, t)=1$ if $w(x, y, z, t)$ $>0$ and $q^{\prime}(x, y, z, t)>0, I_{u}=0$ otherwise; $I_{d}(x, y$, $z, t)=1$ if $w(x, y, z, t)<0$ and $q^{\prime}(x, y, z, t)<0, I_{d}$ $=0$ otherwise; $I_{e}=1-I_{u}-I_{d}$.

In the clear CBL we use potential temperature instead of moisture to obtain " $T$ plumes" or " $w T$ plumes." We cannot compare with Lenschow and Stephens (1980) because the LES of the clear CBL has been performed without moisture. Note that the classification is based on the resolved fields $w, T$, and $q$. This is consistent with the usage of filtered measurements by experimentalists. Local values of the fluxes are computed as sums of grid mean values plus modeled subgrid-scale contributions. The subgrid-scale parts are generally of small importance except for the surface layer. To reduce statistical errors, the results have been averaged over eight (for CBL) or six (for STBL) discrete time points from a time period of $1920 \mathrm{~s}$ (CBL) or $1500 \mathrm{~s}$ (STBL) for which data are available. In these periods the simulations are quasi-stationary. For in- stance, the velocity $\bar{u}$ in the CBL changes by less than $0.5 \mathrm{~m} \mathrm{~s}^{-1}$ in that period within the mixed layer. The averaging periods are sufficiently large in comparison to the convective time scales $\left(z_{i} / w_{*}=510 \mathrm{~s}\right.$ in the CBL and $612 \mathrm{~s}$ in the STBL) to obtain reliable mean values.

For a known indicator function, we can compute the fractional area taken by plumes at height $z$ from

$$
\alpha_{p}=\frac{1}{M_{x} M_{y}} \sum_{i=1}^{M_{x}} \sum_{j=1}^{M_{y}} I_{p}(i, j)
$$

The mean number of plumes per unit length $z_{i}$ in the $x$ direction, e.g., is determined by

$$
N_{x p}=\frac{1}{M_{x} \Delta x} \frac{1}{M_{y}} \sum_{j=1}^{M_{y}} \frac{1}{2} \sum_{i=1}^{M_{x}}\left|I_{p}(i+1, j)-I_{p}(i, j)\right|,
$$

and similarly for $N_{y p}$ in the $y$ direction. Here, the innermost sum counts the number of intersections of a plume surface with a grid line in the computational domain. The outer sum performs the average over all parallel lines and the remaining factors provide the proper normalization. The mean lengths taken by plumes along either of the two horizontal coordinate directions are

$$
d_{x p}=\alpha_{p} N_{x p}^{-1}, \quad d_{y p}=\alpha_{p} N_{y p}^{-1} .
$$

\section{Comparison with experiments}

Several authors have reported conditional mean values based on measurements in clear or cloudy boundary layers to which we can compare the results obtained from our two LES cases. The LES results for the CBL are compared, e.g., in Fig. 3 with data of Young (1988b). The measurements are taken in a cloud-free CBL over land for which $-z_{i} / L$ varied between 11 and 164 (Young 1988a). Hence our simulations $\left(-z_{i} / L=14\right)$ are within the range of parameters covered by these experiments. The analysis distinguishes between updrafts and downdrafts according to "w plumes" with zero threshold values. The area fraction taken by updrafts is smaller than 0.5 in the mixed layer. As a consequence of continuity, the vertical velocity magnitude is larger in updrafts than in downdrafts. This is consistent with a positive skewness of the vertical velocity (Wyngaard 1987; Moeng and Rotunno 1990). If one compares the present results with those obtained by Schumann (1989) from LES of a CBL with zero mean shear, one finds that the shapes of the computed and the measured profiles of the area fraction of updrafts and the corresponding updraft velocities agree closely with each other, in particular in the lower part of the mixed layer. However, the shearfree case gives slightly smaller area fractions in the upper part of the mixed layer (minimum of $\alpha_{u}=0.34$ instead of 0.41 at $z=0.8 z_{i}$ ). Updrafts are warmer than 

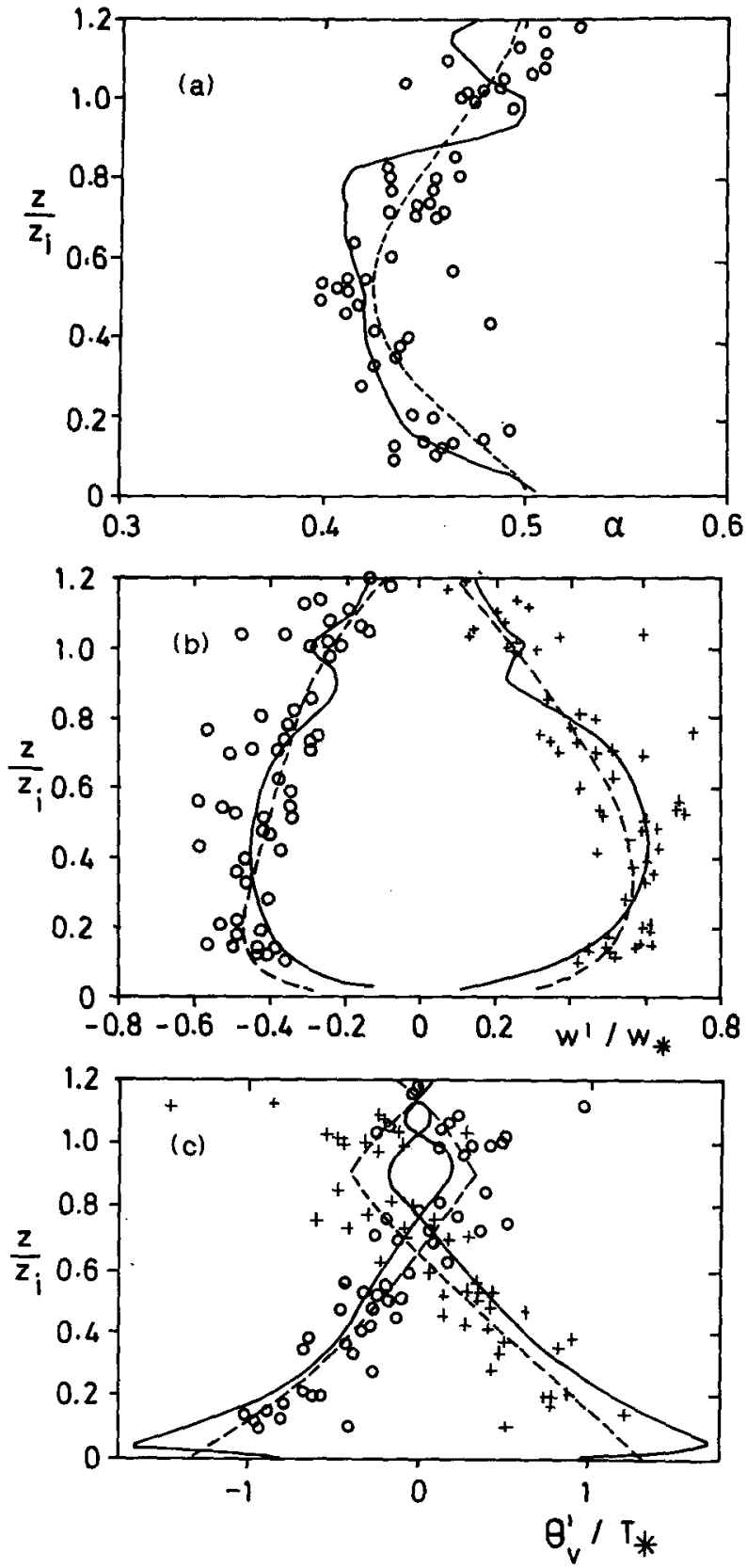

FIG. 3. Comparison of $w$ plume mean values computed from LES for case CBL (full curves) with measured data (symbols) and corresponding interpolations (dashed curves) from Young (1988b). (a) Area fraction of updrafts, (b) updraft and downdraft velocities, (c) potential temperature deviations in updrafts and downdrafts relative to the horizontally averaged potential temperature.

downdrafts, as to be expected, in the mixed layer. The reduction in temperature difference very close to the surface is presumably caused by insufficient resolution of small-scale plumes in the LES at this level. At the inversion, updrafts are rising beyond buoyant equilibrium because of their inertia and this agrees qualitatively with the measurements. At that altitude, the model shows some oscillations which are difficult to explain in detail and are too sharp in the vertical to be detected observationally given the uncertainties in both $z$ and $z_{i}$ as measured by aircraft. Therefore, the differences between measured and computed values are to be considered as small and this confirms the validity of the LES data.

In order to document the spatial parameters of plumes and to identify the possible contributions of rolls, Fig. 4 shows the LES results for profiles of the average diameters of updrafts and downdrafts and the number $N$ of updrafts per unit length, separately for the $x$ and $y$ directions. The plume diameters increase with height above the surface, reach a maximum at midlevels where smaller plumes have merged into larger ones, and then decrease again because the kinematic constraint by the stable layer at the inversion suppresses large-scale vertical motions as does the surface. Moreover, at the inversion, gravity waves with short horizontal wavelengths get mixed with plumes in the present analysis. In the surface layer and at the inversion the plumes have larger widths in the downstream direction because some of the motions are organized as convective roll structures, but at midlevel the diameters are only a little different in the two directions and, hence, most of the large plumes have a circular mean structure with small contributions from downstream rolls. The computed maximum values of the mean diameters are slightly larger than the mean values reported by Young (1988b) for the "width" of updrafts, which ranged from $0.15 z_{i}$ to $0.35 z_{i}$. Young found that the number density of plumes was scattered within a range from 1.4 to 2.8 , and these data agree with the model results to within the scatter of the observations.

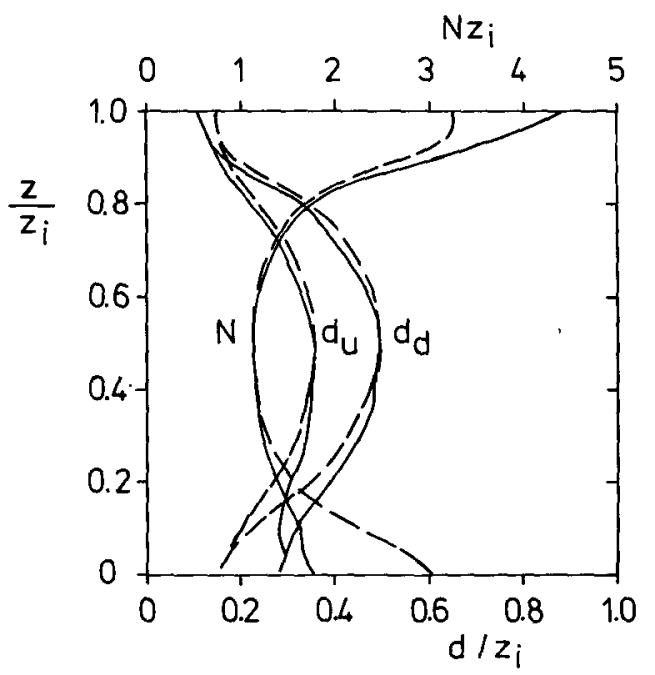

FIG. 4. Mean diameters $d$ of updrafts and downdrafts and number $N$ of updrafts per unit length for case CBL, $w$ plumes. Full curves: in the downstream $x$ direction; dashed curves: in the $y$ direction. 
Different statistics on plumes have been reported by Greenhut and Khalsa (1987) from measurements in a maritime cloud-free CBL. The results shown in Figs. 5 to 7 have been evaluated from the same LES data with their threshold definitions. The computed threshold value profiles reach extreme values $w_{t h}=-0.35 w_{*}$ and $w_{t h}^{+}=0.53 w_{*}$ near $z / z_{i}=0.4$, while Greenhut and Khalsa (1982) and Khalsa and Greenhut (1985) report threshold value profiles for $z / z_{i} \leqslant 0.32$, which are about
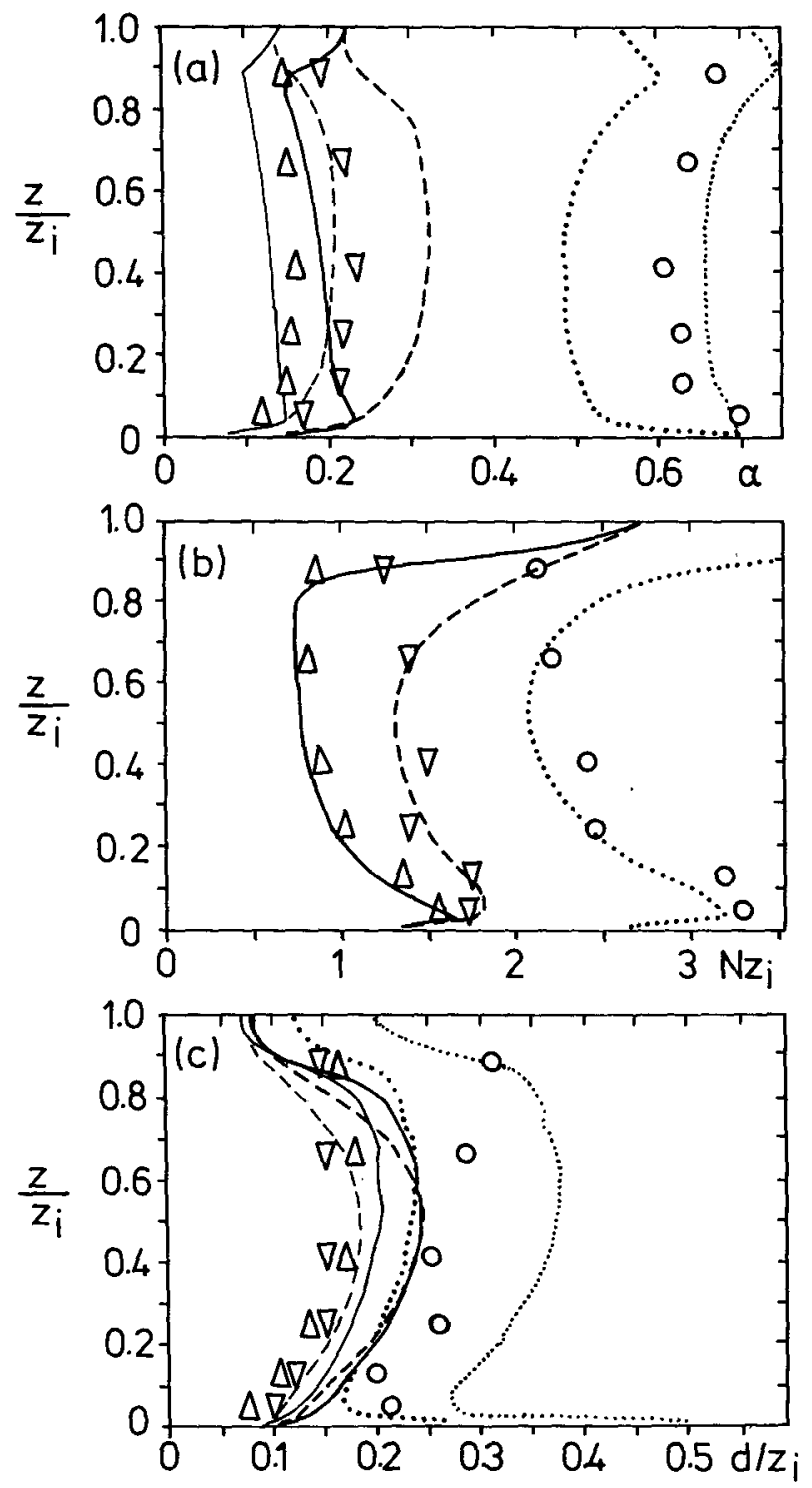

FIG. 5. Comparison of plume mean values computed for case CBL (thick curves without symbols are for the original threshold values, thin curves in some panels are for $\sqrt{2}$ larger threshold values) with measured data (symbols) from Greenhut and Khalsa (1987). Full curves and $\Delta$ : updrafts; dashed curves and $\nabla$ : downdrafts; dotted curves and $O$ : environmental air. (a) Area fraction of plumes, (b) number of plumes per unit length, averaged over both directions, (c) mean diameter of plumes. The thin curves are not included in (b) because they are very close to the thick curves shown.
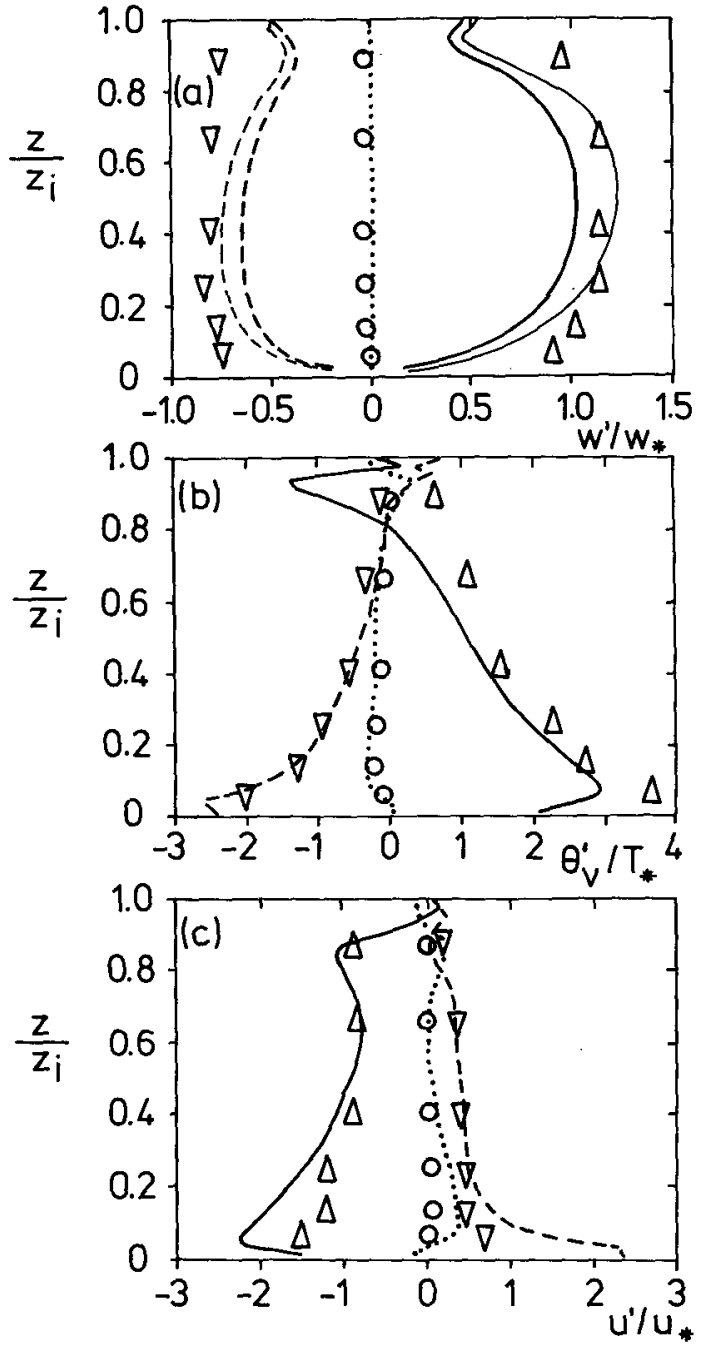

Fig. 6. As in Fig. 5 but for (a) vertical velocity, (b) deviation in virtual potential temperature from the horizontally averaged profile, (c) deviation in wind speed in $x$ direction from average profile.

$10 \%$ to $20 \%$ larger than the LES results, in particular at small heights. Such an underestimate is reasonable because the LES values are computed from the resolved fields using horizontal grid spacings of about $52 \mathrm{~m}$, while the experimental data are obtained with a resolution smaller than $10 \mathrm{~m}$. The agreement between measured and computed data is generally good, but even better agreement is obtained if we use threshold values that are larger by a factor of $\sqrt{2}$. The results shown in Figs. 5 to 7 are much different from Young's results and those reported by Lenschow and Stephens (1980) because of the different criteria used.

Figure 5 shows that updrafts with large vertical velocity occupy only about $20 \%$ of the total area. Their diameter increases from the surface up to the upper third of the mixed layer. The number of updrafts per unit length is smaller than the number of downdrafts. 


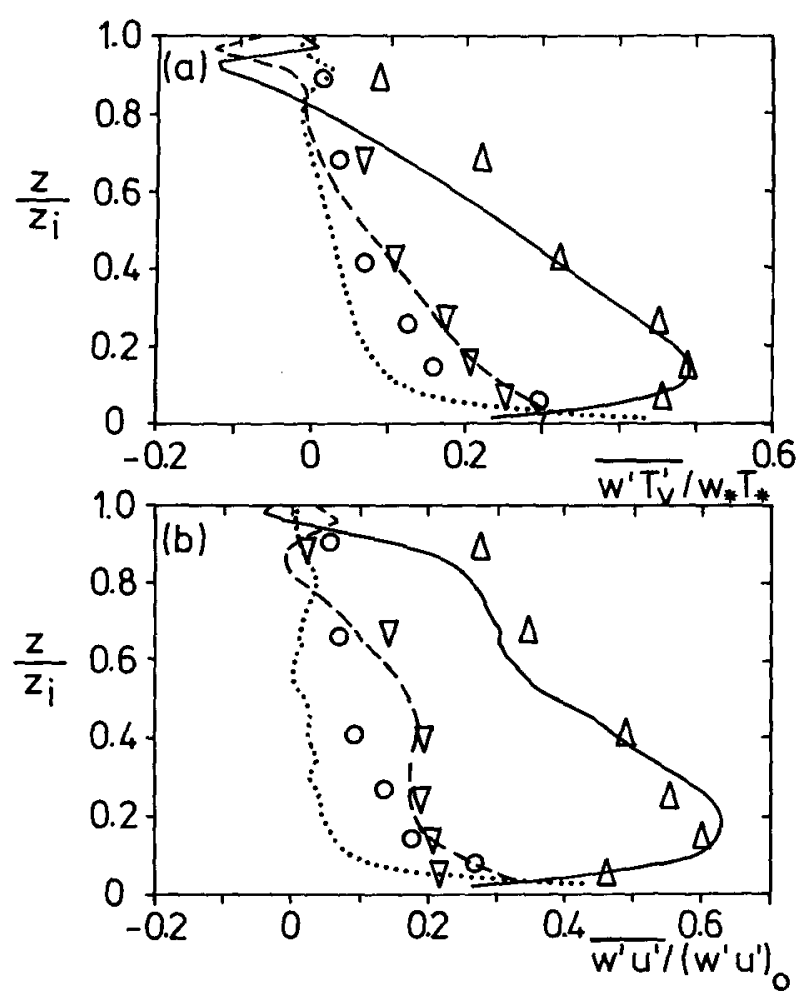

FIG. 7. As in Fig. 5 but for (a) vertical buoyancy flux, (b) momentum flux, normalized by the surface fluxes.

The downdrafts occupy a little larger area fractions and have greater frequency while diameters of updrafts and downdrafts are roughly equal. The quantitative agreement with the observations is reasonable but even better with the larger thresholds, for which the area fractions and diameters of updrafts and downdrafts are smaller and very close to the observations. The updraft velocity, Fig. 6 , is larger than the downdraft velocity and these values are larger in the criterium of Greenhut and Khalsa than in that of Young. The profiles shown have about twice the magnitude of the threshold profiles, and this agrees with the observations (G. K. Greenhut, personal communication 1990). But the LES underestimates the absolute values of the vertical velocities in the present analysis by about $20 \%$ (the quantitative agreement with observations is again better when using larger thresholds). The temperature values are quite close to the observations (this result is rather insensitive to the threshold values), as are the conditionally averaged downstream velocity fluctuations when normalized by the friction velocity. Updrafts carry most of the momentum flux. This can be seen from the larger difference in horizontal velocity shown in Fig. $6 \mathrm{c}$ and in the larger flux values from updrafts shown in Fig. 7b. Figure 7a shows that also most of the vertical heat flux is carried by the strong updrafts. The LES flux results for updrafts and downdrafts are quite insensitive with respect to the actual threshold value, but the agreement between fluxes from the environmental regions was better when using larger threshold values. Overall, the normalized profiles agree with those of Greenhut and Khalsa (1987) to a large degree in spite of the fact that details of forcing by shear and surface friction are different. The agreement is better if we use larger threshold values, and this is reasonable if one considers the different filters that have been applied to the data.

Larger differences are found in Fig. 8, where LES data are compared with data of Nicholls (1989), for a STBL. The LES data, given by the two sets of curves, refer to downdrafts defined as $w$ plumes with $w_{t h}=0$ or $w_{\text {th }}^{\bar{b}}=-0.5 w_{* D}$. Nicholls' sampling method differs from ours. In the time series of the vertical velocity field he first searched for the event that has $w^{\prime}$ $<-0.5 w_{* D}$. He then used the nearest zero crossing points on either side of that event to define the extent of the downdraft and its intersection width. As a consequence, Nicholls' method selects fewer downdrafts than we obtain for $w_{t h}^{-}=0$ but more than for $w_{t h}^{-}$ $=-0.5 w_{* D}$. Mainly because of the difference in the sampling procedure, the agreement is poorer for the STBL than for the clear case. But the differences also certainly arise from deficiencies in the LES. The measurements indicate clearly that the measured downdrafts are of smaller diameter and larger frequency than in the simulations. This is probably caused by a still too coarse horizontal resolution in the LES for this STBL case. The ratio $\Delta x / z_{i}$ amounts to 0.123 in the
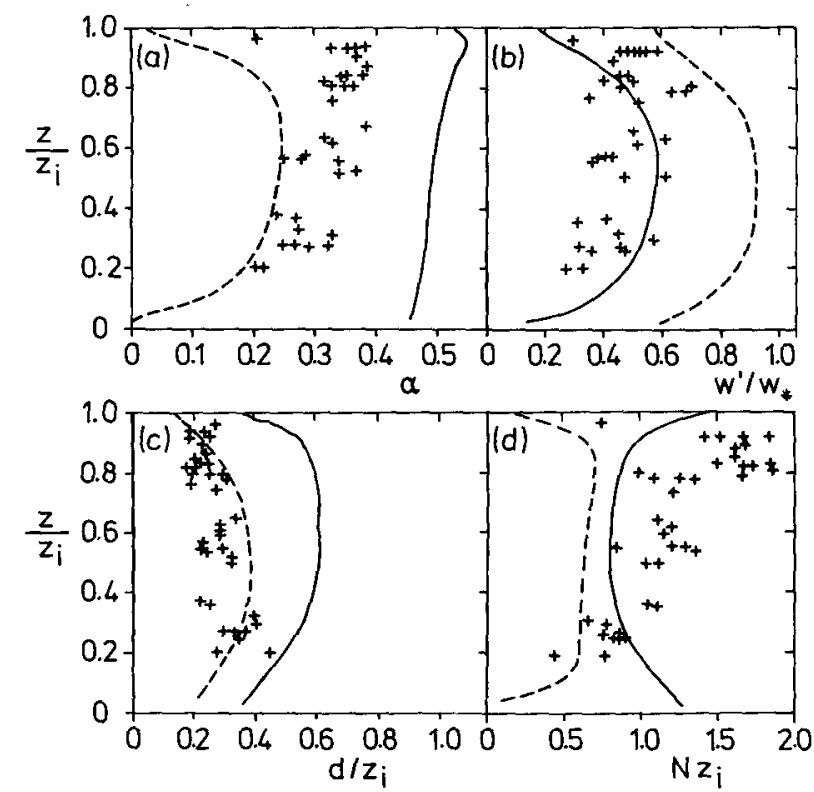

FIG . 8. Computed values from LES for case STBL using $w$ plumes (full curves: $w_{t h}^{-}=0$; dashed curves: $w_{t h}^{-}=-0.5 w_{* D}$ ), with measured data (symbols) from Nicholls (1989) (with a nonlocal plume definition). (a) Area fraction of downdrafts, (b) downward vertical velocity averaged over the downdrafts, (c) diameter of downdrafts in $y$ direction, (d) number of downdrafts per unit length in $y$ direction. 
STBL, while it is 0.0506 in the CBL simulations. Hence, a diameter of about $0.25 z_{i}$, as found in the measurements, corresponds to only two horizontal grid cells in the simulation of the STBL. For zero threshold values, Fig. $8 \mathrm{c}$ shows that the maximum normalized downdraft diameters are about $20 \%$ larger than in the CBL, compare Fig. 4. However, differences will also be caused by different buoyancy forcing. Nicholls' cases are driven by top cooling with very little surface heating. Some of his data were obtained for cloudy mixed layers that were dynamically decoupled from a surface layer. Hence, we cannot expect strict quantitative agreement in this comparison.

Next we compare results obtained from case STBL for $w q$ plumes. Such plumes were considered before by Nicholls and LeMone (1980) and by Penc and Albrecht (1987). Here we compare to data obtained by the latter from measurements in a maritime boundary layer with broken or solid stratus clouds without a decoupled subcloud layer. Penc and Albrecht show data for the rms values of the vertical fluctuations, $w^{\prime} / w_{* D}$, from their own analysis and from Nicholls and LeMone (1980). In the mixed layer, the computed results (the variance is shown in Fig. 2f) vary between 0.6 to 0.7 and fall within the range of the measurements that vary between 0.5 and 0.9. In Fig. 9, we compare with respect to area fractions of the three classes of $w q$ plumes. Penc and Albrecht (1987) reported average-area fractions of 0.28 to 0.29 for updrafts, 0.27 to 0.31 for downdrafts, and 0.41 to 0.44 for environmental air from measurements for various cases. R. S. Penc (personal communication 1990) provided the data plotted in Fig. 9. We see that our LES results show little variation with altitude. The computed results for the area fractions reveal about equal fractions of the three plume categories, while the data of Penc and Albrecht suggest that updrafts are concentrated in slightly smaller areas. In

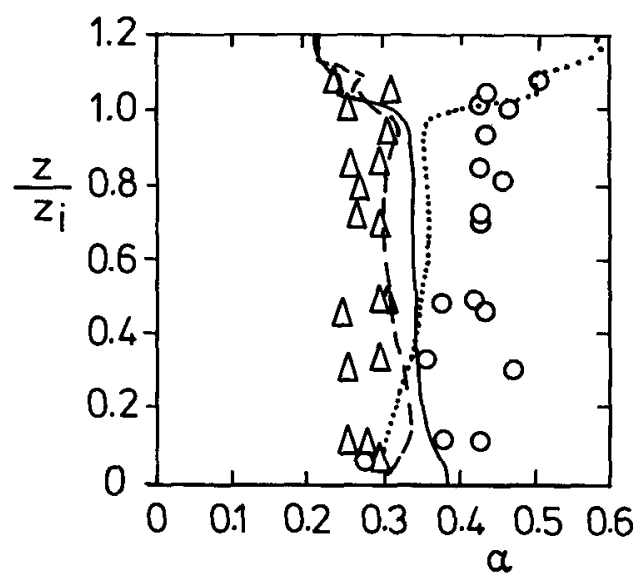

FIG. 9. Area fractions of updrafts (full curve), downdrafts (dashed), and environmental air (dotted) in a STBL for $w q$ plumes. The symbols represent measurements, $\Delta$ for updrafts, $O$ for environmental regions. any case, because of using the same sampling scheme, the agreement is better than in Fig. 8.

Figure 10 shows differences between updraft and downdraft moisture and temperature profiles. Updrafts have larger moisture than downdrafts because that is a condition entering the criterion of $w q$ plumes. In the lower part of the boundary layer this difference is caused by surface evaporation. The difference decreases with increasing height because of lateral mixing between updrafts and downdrafts. The moisture difference becomes very large at the inversion because downdrafts from above the inversion carry very dry air. The magnitude of this difference reflects the strong vertical decrease in the mean moisture profile at this level, see Fig. 2. Because of surface heating, the updrafts are warmer than downdrafts for most of the mixed layer by up to six units of the convective temperature scale in rough agreement with the results for the dry CBL, see Fig. 6b. In the stratus layer, water condenses, resulting in positive differences in liquid water content between updrafts and downdrafts. The latent heat release causes additional buoyancy forcing of the circulation as revealed by the differences in virtual potential temperature. But the updrafts are colder than downdrafts at the inversion because updrafts rise beyond the level of zero buoyancy (virtual temperature difference) into the much warmer air above the inversion, see Fig. 2. The differences between temperatures of updrafts and downdrafts would certainly be smaller for a less stable inversion.

The data points in Fig. 10 are taken from Penc and Albrecht (1987). The absolute values of the data differ considerably from the simulation results because of differences in the absolute values of the fluxes and the changes in mean temperature and moisture at the inversion. Therefore, the data have been normalized and plotted according to the upper ordinate in Fig. 10. Here we use the convective scales of temperature $T_{* D}$ and moisture $q_{* D}$ such that $w_{* D} T_{* D}$ equals the mean vertical temperature flux and $w_{* D} q_{* D}$ the mean vertical moisture flux averaged from $z=0$ to $z=z_{i}$, where $w_{* D}$ is Deardorff's velocity scale, see (5). From the data of flights 4 and 5 analyzed by Penc and Albrecht (1987) we determine $w_{* D}=0.54,0.31 \mathrm{~m} \mathrm{~s}^{-1}, T_{* D}$ $=0.007,0.0023 \mathrm{~K}, q_{* D}=0.016,0.011 \mathrm{~g} \mathrm{~kg}^{-1}$, for the two flights, respectively. In the LES results, the corresponding scales are $T_{* D}=0.027 \mathrm{~K}, q_{* D}=0.017 \mathrm{~g}$ $\mathrm{kg}^{-1}$. The normalized data agree quite well with the simulated results. This is true in particular with respect to the liquid water and total moisture differences. The subcloud temperature differences show larger deviations but the measurements support the strongly negative temperature difference at the inversion. In the subcloud layer, the results are of a magnitude that is comparable with data recorded by Nicholls and LeMone (1980).

These results show that the simulations for the CBL agree quantitatively with observations from very dif- 


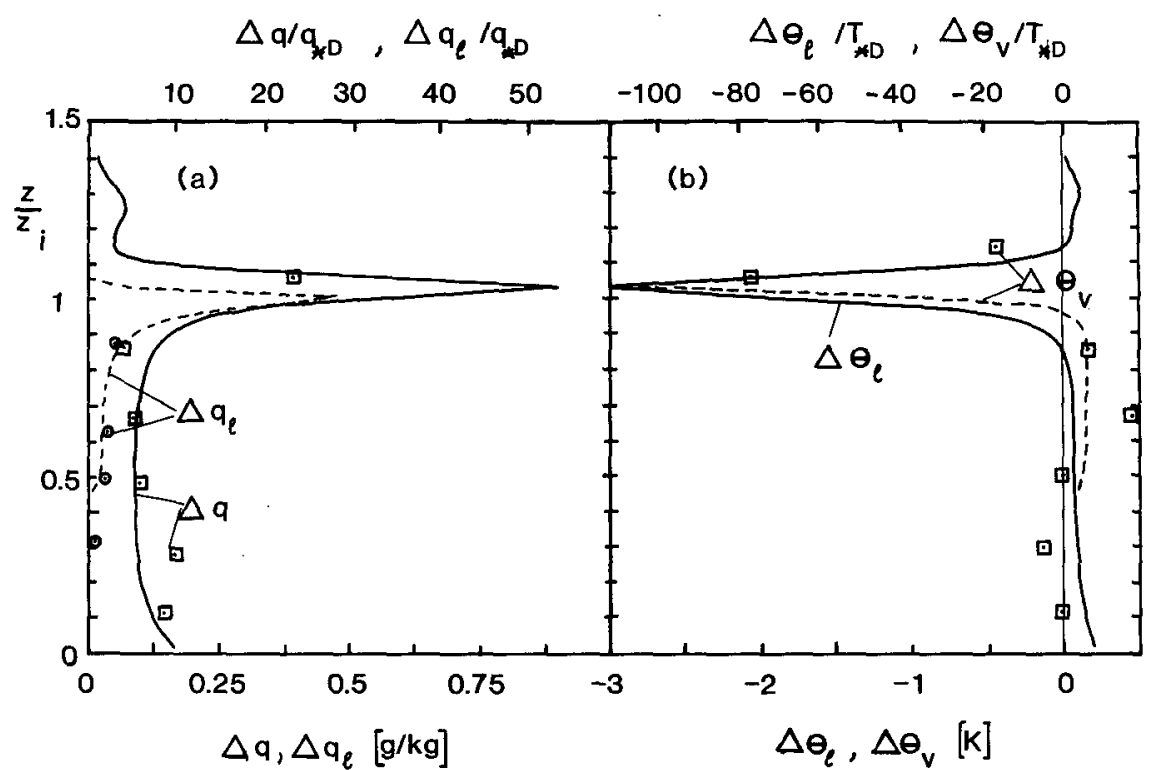

FIG. 10. Differences between mean values in updrafts and downdrafts for $w q$ plumes in a STBL as computed (curves) and measured by Penc and Albrecht (1987) (circles from flight 4, squares from flight 5 ). The measured data refer to the upper normalized ordinate. (a) Differences in total $(\Delta q)$ and liquid water content $\left(\Delta q_{l}\right),\left(\right.$ b) differences in liquid water potential temperature $\left(\Delta \theta_{l}\right)$ and virtual potential temperature $\left(\Delta \theta_{v}\right)$.

ferent field experiments and analysis methods so that we can use the LES data to determine the parameters of Eqs. (1)-(4). For the STBL, the agreement is still sufficient to identify changes in these parameters as introduced by a cloud layer.

\section{Top-hat profile parameters}

Horizontally averaged flux values versus height (full curves) for various transported quantities are shown in Fig. 11 for the CBL and in Fig. 12 for the STBL. Also plotted are the contributions from plumes according to the simple top-hat approximation:

$$
\overline{w^{\prime} f^{\prime}} \approx\left(\alpha_{u} w_{u} f_{u}+\alpha_{d} w_{d} f_{d}+\alpha_{e} w_{e} f_{e}\right) .
$$

The various curves apply to different types of plume definitions but all with zero threshold values. In general, the top-hat approximation underestimates the actual flux profiles, which means that the coefficient $a$ in Eq. (3) should be larger than unity. The strong reduction of the approximated fluxes in the lowest $10 \%$ of the boundary layer is caused by insufficient resolution of the narrow plumes near the surface. Here, and only here, large portions of the total fluxes in the LES are carried by subgrid-scale motions.

From Fig. 11, for the CBL, we see that $w$ plumes

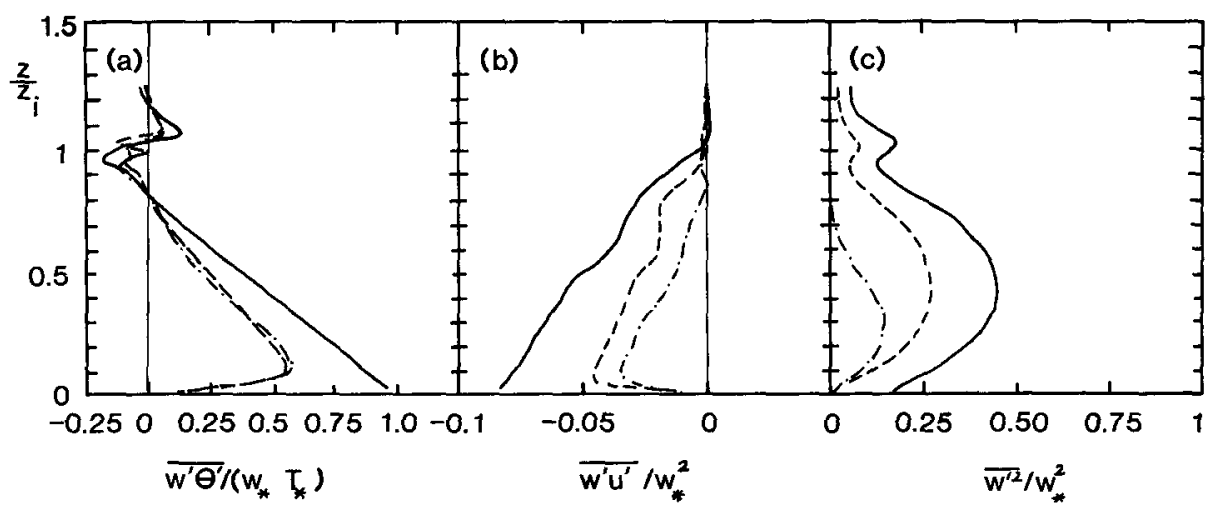

FIG. 11. Normalized fluxes in the CBL. (a) Heat flux, (b) momentum flux, (c) vertical velocity variance. Full curves: total flux; dashed curves: top-hat profile approximation for $w$ plumes, dash-dotted curves: same for $T$-plumes. 

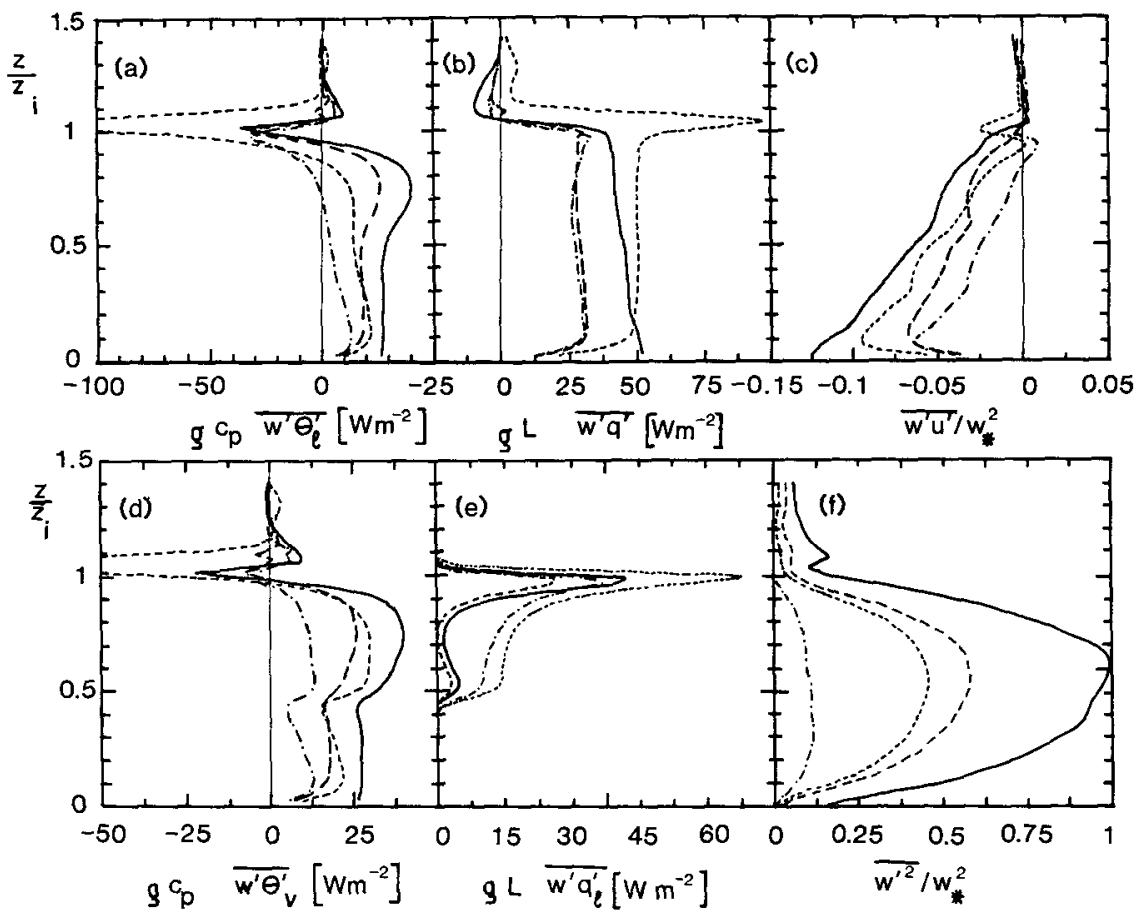

FIG. 12. Fluxes in the STBL. (a) Heat flux, (b) latent heat flux, (c) momentum flux, (d) buoyancy flux, (e) liquid water flux, (f) normalized vertical velocity variance. Full curves: total flux, long-dashed curves: top-hat profile approximation for $w$ plumes, dash-dotted curves: same for $q$ plumes, short-dashed curves: same for $w q$ plumes.

give about the same contributions from top-hat profile approximations as do $T$ plumes with respect to the temperature flux; but $T$ plumes underestimate plume contributions to the momentum flux and to vertical velocity variance, in particular in the upper part of the boundary layer. $T$ plumes do not describe the fluxes as well as $w$ plumes because in the upper boundary layer the correlation between $w^{\prime}$ and $T^{\prime}$ is weak, and therefore not much of the variance in $w^{\prime}$ is associated with events defined in $T^{\prime}$. However, we see that the $w$ plume results are uniformly smaller than the actual fluxes by a factor of order 0.6 and close to constant throughout the mixed layer $0.1 \leqslant z / z_{i} \leqslant 0.8$. This is the first indication that $w$ plumes provide more uniform top-hat approximations to fluxes than other types of plume definitions.

Figure 12 shows similar comparisons for the STBL, including fluxes of nonconserved quantities like buoyancy or liquid water. Generally, the top-hat approximation, Eq. (10), underestimates the actual flux magnitudes. However, it overestimates the moisture fluxes, given in Fig. 12, panels (b) and (e), and the heat and buoyancy fluxes, (a) and (d), when using $q$ or $w q$ plumes. Especially the $w q$ plumes (short-dashed curves) give much too large moisture flux at the inversion. This is partly a consequence of the criterion that selects those plumes with large correlation between moisture and vertical velocity. The overestimate is particularly large at the inversion where updrafts are very much more moist than downdrafts, see Fig. 10a, so that the related fluxes are large. Also, dry air entrained from above the inversion exhibits large negative moisture fluctuations correlated with negative vertical velocity resulting again in a strong positive vertical flux. From the numerical results we found that each of these two positive fluxes is much larger than the total flux and the negative contribution by the environmental part is too weak to balance the overestimates from updrafts and downdrafts. A smaller overestimate might arise at less stable inversions. The $w q$ plumes also overestimate the entrainment heat flux because entrained air exhibits negative moisture fluctuations but positive temperature deviations. The $q$ plumes (dash-dotted curves) result in flux profiles that differ qualitatively from the actual flux profiles not only at the inversion but also in the mixed layer. Obviously, only $w$ plumes (long-dashed curves) give uniform top-hat approximations. This is reasonable because $w$ plumes are defined according to the vertical velocity, which is responsible for vertical transports by plume motions.

In Fig. 13, we show the coefficients $a$ and $b$ and the flux velocity $\omega^{*}$, which result from Eqs. (1)-(4) using the LES results for $w$ plumes. Profiles of $\omega^{* *}$ are not plotted because, by definition, $\omega^{* *} / \omega^{*}=\alpha_{d} \approx 0.5$ for plume definitions that distinguish between updrafts and downdrafts only. In this case, $\alpha_{e}=0$, and continuity 

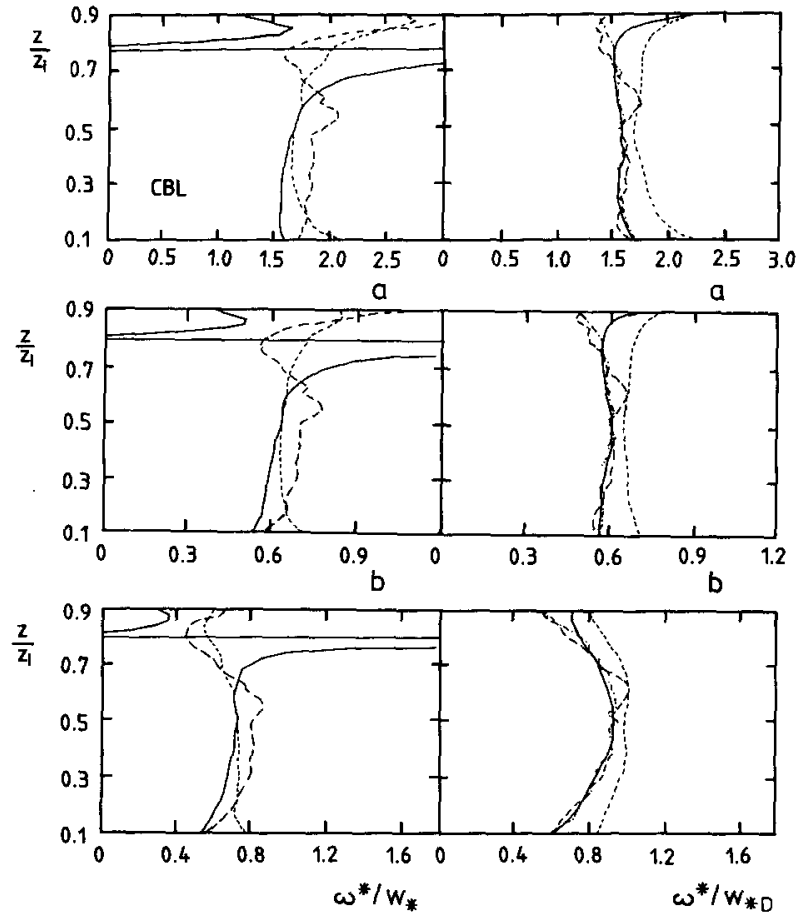

FIG. 13. Coefficients $a$ (top panels) and $b$ (middle) and flux velocity $\omega^{*}$ (bottom), as defined in Eqs. (1), (3), (4), for $w$ plumes with zero threshold values. Left: CBL, right: STBL. The various curves result from different values of the transported quantity $f$. Solid: $\theta$ (or $\theta_{l}$ for STBL), long dashed: $u$, short dashed: $w$, dash-dotted: $q$.

implies $\alpha_{d} \omega^{*}=\omega^{* *}=a \alpha_{u} w_{u}=b \sigma_{w}$. The results are shown only for $z>0.1 z_{i}$ because the LES underestimates contributions from narrow plumes near the surface. This underestimate causes a strong increase in the coefficients near the surface, while Businger and Oncley (1990), from high-resolution data, find an almost constant value $b \approx 0.6$ even at very small altitudes. In Fig. 13, the flux velocities for the STBL are normalized by $w_{* D}$, Eq. (5). Penc and Albrecht (1987) proposed to normalize by $\left(w_{* D}^{2}+4 u_{*}^{2}\right)^{1 / 2}$. However, this normalization gives only small differences because of the smallness of $u_{*} / w_{* D}$ in the present simulations.

We find that the coefficients $a$ and $b$ are not strictly constant and not strictly independent of the transported quantity. The differences between the various coefficients and flux velocities are smaller if one compares the results only for the scalars $\theta\left(\theta_{l}\right)$ and $q$, while for the velocity components larger differences appear. This might be caused by pressure forces that affect the latter more than scalar quantities. The results show large fluctuations in the upper part of the mixed layer because both the fluxes and the plume differences become small or even change sign there, but not exactly at the same altitude. Hence, the top-hat approximation is generally meaningful only in the mixed layer; i.e., for $z / z_{i} \leqslant 0.8$. Much larger variations have been found in comparable plots (not shown) for $q$ plumes ( $T$ plumes) and $w q$ plumes ( $w T$ plumes). The differences between the various curves are smaller in the STBL than in the $\mathrm{CBL}$. This might originate from the fact that the fluxes in the STBL in the upper mixed layer are larger than those of the CBL, so that the coefficients and flux velocities are determined with less uncertainty. As can be seen from Eqs. (1)-(4), the ratio between $a, b$, and $\omega^{* *}$ depends only on the plume properties, independent of the quantity $f$.

The largest degree of uniformity of the coefficients and flux velocities for the various transported quantities has been found for $w$ plumes with threshold values as defined by Greenhut and Khalsa (1987). Further evaluations of the mixed layer of the CBL in $w$ plumes with such threshold values as used by Greenhut and Khalsa (1987) result in $a \approx 1.3 \pm 0.1, b \approx 0.4 \pm 0.1$, $\omega^{*} / w_{*} \approx 0.4 \pm 0.1$. Figure 14 shows, e.g., the LES results for $\omega^{* *}$ in $w$ plumes in comparison with experimental data for the CBL. The magnitude of $\omega^{* *}$ / $w_{*} \approx 0.24 \pm 0.05$ is within the range of data reported by Betts (1976) and Nicholls and LeMone (1980) who considered $w q$ plumes. Using $\sqrt{2}$ larger threshold values result in about $15 \%$ smaller flux velocities.

\section{Conclusions}

The LES results compare generally well with observations. For the CBL the only significant differences are in the near surface layer (where subgrid-scale contributions are important) and near the inversion where turbulence interacts with gravity waves and where the model shows variations at vertical scales, which are too small to be detected observationally given the uncertainties in both $z$ and $z_{i}$ as measured by aircraft. Obviously, the LES method has become a validated tool to predict properties of convective boundary layers. The differences between observed and simulated STBLs

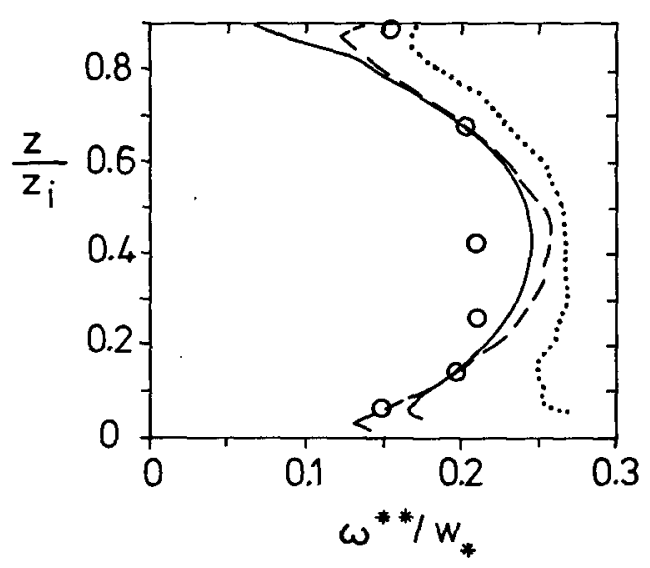

FIG. 14. Flux velocity $\omega^{* *} / w_{*}$, see Eq. (2), for $w$ plumes with nonzero threshold values as defined by Greenhut and Khalsa (1987), for the CBL. The circles indicate data from these authors. Solid: $f$ $=\theta$, dashed: $u$, dotted: $w$. 
are considerably larger than those between observed and simulated CBLs. This may result from one or a combination of the following reasons: differences in the sampling scheme used in analyzing the STBLs, the coarser grid resolution for the STBL simulation, differences in the actual inversion properties, or effects of physical processes not included in the LES of the STBL (e.g., solar radiation). The CBL simulations cover both the properties of the clear CBL over land and over sea. Hence, physical processes other than the surface heat and moisture fluxes are of little importance for convective boundary layers. The results match very well with the well-established convective scales.

We have shown that top-hat approximations give a most uniform representation of the vertical profile of fluxes of various transported quantities if the plumes are $w$ plumes, i.e., classified according to the local vertical velocity. The approximation is generally better for nonzero threshold values, but this requires one to treat environmental regions in addition to updrafts and downdrafts. Classifications based on temperature or humidity give uniform approximations only if these quantities are well correlated with vertical velocity fluctuations. The combined usage of vertical velocity fluctuations with temperature or humidity fluctuations can result in overestimates of the fluxes, in particular near strong inversion. For $w$ plumes, the top-hat approximation gives a fair approximation not only for scalar fluxes but also for fluxes of horizontal momentum and for the vertical velocity variance.

For $w$ plumes with zero threshold values, and for the mixed layer of the CBL, we recommend $a \approx 1.6$ $\pm 0.1, b \approx 0.6 \pm 0.1$. Hence, the simple top-hat approximation, Eq. (10), describes $a^{-1} \approx 0.625 \pm 0.04$ or about $60 \%$ of the total fluxes of all relevant quantities. The same result has been found by Young (1988b). The remaining $40 \%$ of the fluxes are transported by subplume motions that are lost when averaging over updrafts and downdrafts separately. The subplume fraction is smaller ( $10 \%$ to $30 \%$ in the LES) when using $w$ plumes with nonzero thresholds, where environmental regions are treated separately, as also shown by Khalsa and Greenhut (1985). For $w$ plumes with zero thresholds, the value of $\omega^{*} / w_{*}$ is of order 0.7 and weakly dependent on $z / z_{i}$. For $w$ plumes with nonzero thresholds as in Greenhut and Khalsa (1987), we support their result $\omega^{* *} / w_{*}=0.24 \pm 0.05$ within the mixed layer of a CBL. In a subsequent paper, we will consider budgets of various fields within plumes.

Acknowledgments. We are grateful to T. Hauf, S. J. S. Khalsa, R. S. Penc, G. S. Young, and the reviewers for useful discussions and comments on an earlier version of this paper. This work was started while the first author was a visiting scientist at the National Center for Atmospheric Research, for which he acknowledges financial support. The National Center for
Atmospheric Research is sponsored by the National Science Foundation.

\section{REFERENCES}

Betts, A. K., 1973: Nonprecipitating cumulus convection and its parameterization. Quart. J. Roy. Meteor. Soc., 99, 178-196.

__ 1976: Modeling subcloud layer structure and interaction with a shallow cumulus layer. J. Atmos. Sci., 33, 2363-2382.

Businger, J. A., and S. P. Oncley, 1990: Flux measurements with conditional sampling. J. Atmos. Oceanic Technol., 7, 349-352.

Deardorff, J. W., 1980: Stratocumulus-capped mixed layers derived from a three-dimensional model. Bound-Layer Meteor., 18, 495-527.

Greenhut, G. K., and S. J. S. Khalsa, 1982: Updraft and downdraft events in the atmospheric boundary layer over the equatorial Pacific Ocean. J. Aimos. Sci., 39, 1803-1818.

- - and - 1987: Convective elements in the marine atmospheric boundary layer. Part I: Conditional sampling statistics. J. Climate Appl. Meteor., 26, 813-822.

Khalsa, S. J. S., and G. K. Greenhut, 1985: Conditional sampling of updrafts and downdrafts in the marine atmospheric boundary layer. J. Atmos. Sci., 42, 2550-2562.

Lenschow, D. H., and P. L. Stephens, 1980: The role of thermals in the convective boundary layer. Bound.-Layer Meteor., 19, 509532.

Manton, M. J., 1975: Penetrative convection due to a field of thermais. J. Atmos. Sci., 32, 2272-2277.

Moeng, C.-H., 1984: A large-eddy-simulation model for the study of planetary boundary-layer turbulence. J. Atmos. Sci., 41, 20522062.

- 1986: Large-eddy simulation of a stratus-topped boundary layer. Part I: Structure and budgets. J. Atmos. Sci., 43, 2886-2900.

, and J. C. Wyngaard, 1989: Evaluation of turbulent transport and dissipation closures in second-order modeling. J. Atmos. Sci., 46, 2311-2330.

_- and R. Rotunno, 1990: Vertical-velocity skewness in the buoyancy-driven boundary layer. J. Atmos. Sci., 47, 1149-1162.

—_, and U. Schumann, 1990: Composite updraft and downdraft in stratus-topped boundary layer. Proc. Ninth Symp. on Turbulence and Diffusion, Roskilde, Denmark, Amer. Meteor. Soc., $7-10$.

Nicholls, S., 1989: The structure of radiatively driven convection in stratocumulus. Quart. J. Roy. Meteor. Soc., 115, 487-511.

- - , and M. A. LeMone, 1980: Fair weather boundary layer in GATE: the relationship of subcloud fluxes and structure to the distribution and enhancement of cumulus clouds. J. Atmos. Sci., 37, 2051-2067.

Nieuwstadt, F. T. M., 1990: Direct and large-eddy simulation of free convection. Proc. Ninth Int. Heat Transfer Conf., Jerusalem, Amer. Soc. Mech. Eng., 37-47.

Penc, R. S., and B. A. Albrecht, 1987: Parametric representation of heat and moisture fluxes in cloud-topped mixed layers. Bound.Layer Meteor, 38, 225-248.

Schmidt, H., and U. Schumann, 1989: Coherent structure of the convective boundary layer derived from large-eddy simulations. J. Fluid Mech., 200, 511-562.

Schumann, U., 1989: Large-eddy simulation of turbulent diffusion with chemical reactions in the convective boundary layer. Atmos. Environ., 23, 1713-1727.

Wyngaard, J. C., 1987: A physical mechanism for the asymmetry in top-down and bottom-up diffusion. J. Atmos. Sci., 44, 10831087.

Young, G. S., 1988a: Turbulence structure of the convective boundary layer. Part I: Variability of normalized turbulence statistics. $J$. Atmos. Sci., 45, 719-726.

_ 1988b: Turbulence structure of the convective boundary layer. Part II: Phoenix 78 Aircraft observations of thermals and their environment. J. Atmos. Sci., 45, 727-735. 